\title{
Studying the applicability of non-destructive techniques in diagnosing defects of soil-cement columns
}

\author{
Tham Hong Duong*
}

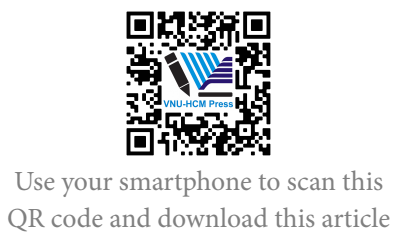

\begin{abstract}
This article studies the applicability of the Non-Destructive Techniques (NDT) into semi-rigid structures, particularly in soil-cement columns. A numerical model for the semi-rigid soil-cement column is first created. Different kinds of defects are intentionally allocated in the model, including necking, bulging, and degraded stiffness. The assumption is that by using an excitation as an impact load at the structure head and then letting the structure vibrating freely, studying the wave characteristics inside the structure, i.e., responsive velocity and displacement at various points along the column shaft, the impedance could be determined. If there is any variation in the mechanical impedance $Z$, which is defined to be the product of mass density, area of the cross-section, and the wave velocity, the defects are confirmed. The shape of the impedance curves with respect to combined defects is analyzed, and spectral response curves are plotted. The process of analysis in the time domain and frequency domain for the soil-cement column is conducted using Fast Fourier Transformation. The theoretical and computed impedance of the structure from the numerical model will be compared with each other, in the shape of the responsive curves, and the distinguished issues; some discussions on the propagation of waves through semi-rigid structures are summarized. There is no distinguishing feature in the characteristics of the impedance of the structure revealed. It comes to the conclusion that the applicability of the vibration test is not clearly recognized. There is quite a difficulty in evaluating the performance of the semi-rigid structures like soil-cement columns by using vibration or impact load test. This outcome suggests that the column is not the same as the pile, and another alternative and/or approach is recommended to apply in quality assurance/control QA/QC for such embedded semi-rigid structures.

Key words: Defects, Non-Destructive Test, Mechanical Impedance, Soil-Cement Column
\end{abstract}

Ho Chi Minh City Open University

\section{Correspondence}

Tham Hong Duong, Ho Chi Minh City Open University

Email: tham.dh@ou.edu.vn

History

- Received: 2020-09-30

- Accepted: 2020-12-31

- Published: 2020-12-31

DOI : 10.32508/stdj.v23i4.2474

\section{Check for updates}

\section{Copyright}

(c) VNU-HCM Press. This is an openaccess article distributed under the terms of the Creative Commons Attribution 4.0 International license.

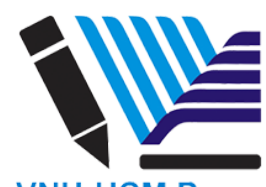

\section{INTRODUCTION}

Soil-Cement Columns has gradually proved to be a satisfactory solution for a deep foundation. The soil now plays the role of being a material for the purpose of supporting the gravity load from the superstructure. The quality of this kind of structure depends on many factors, including the quality of the material ingredients at the site, the technology of mixing, the depth of work ability, soil stratification, and others, etc. Without any transmission from the shaft to the outer medium, this structure cannot be called the "pile," and the structure is uniquely different as compared to that of a pile. So testing the integrity of a soil-cement column is vital.

Because it is mixed at the site, the columns may have some defects. Meanwhile, so many techniques such as Pile Integrity Test (PIT), Impedance Log Technique (IL), Cross-hole Sonic Logging (CSL), etc. ${ }^{1}$ are successfully applied to pile foundation; the techniques appear to be irrelevant for this kind ${ }^{2}$. Combined with uncertainties in signal data processing and software (epistemic), and others on the side of nature (aleatory), it is actually a complex task of the quality assessment for this semi-rigid structure.

Several common questions for this structure are how to assess the quality of the material and what is the most typical factor for evaluating the strength or the rigidity of this semi-rigid structure?

This article would study the possibility of applying the techniques which are well-applied to pile into a soilcement column for detecting the defects inside it.

\section{BACKGROUND ABOUT PILE TESTS}

The most important role of structural health monitoring (SHM) is to obtain information about the health of the structure, to early detect the damage or defects in the structure and give feedback about the possible failures in the future; some assessments on the potential of time-dependent failure also are suggested using quantitative evidence. If there is no information withdrawn from the test, the test is useless and impractical. There are numerous methods of testing the structures. It depends on which kind of damage and the purpose, 
then the method is selected. For instance, for detecting the defects, the integrity test will be applicable in measuring the change in the impedance of the structure. For the pile and columns, there are commonly five popular kinds of defects which are possibly occurred to a soil-cement column: Necking, Bulging, Void, Discontinuity (i.e., Crack or Soil intrusion), and low quality of the mixing product. The two following methods will be applied to check the applicability of the approach for a soil-cement column in this study.

\section{Testing method and Signal analysis}

As mentioned in ${ }^{3}$, most of the tests are developed according to two main categories of concepts: Reflection and Direct Transmission. Impulse Response (IR), Transient Dynamic Response (TDR), Sonic Echo (SE), Impedance Log (IL), and Impact Echo (IE) is of the former; and Cross-hole Sonic Logging (CSL) and Parallel Seismic (PS) tests are of the latter. People use CSL to check the diameter of the embedded bored pile at the site.

\section{Parallel Seismic Test}

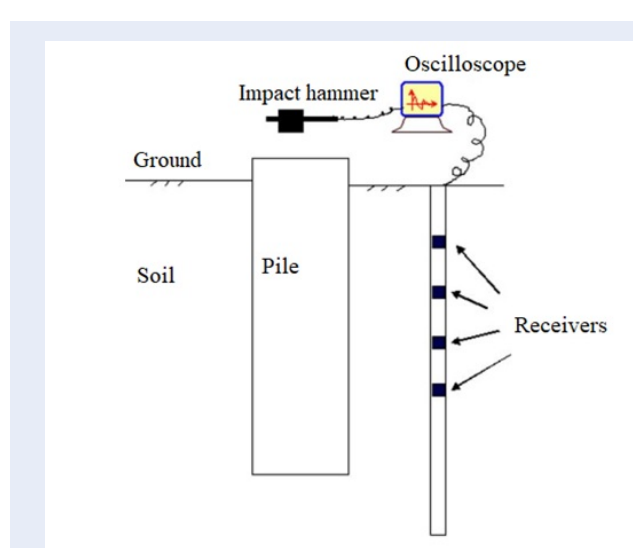

Figure 1: Parallel Seismic test.

This method is developed to evaluate the geometric configuration of the concrete pile, as in Figure $\mathbf{1 .}$ Other purposes could be attained, such as Diagnosing the embedded defects and indirectly providing the data for determining the pile's bearing capacity.

\section{Impedance Method}

The idea is that the stress wave propagating through an elastic medium will be analyzed using the solution of the second-order partial differential equation as in Equation (1) (Figure 2) below:

$$
V_{p}^{2} \frac{\partial^{2} u}{\partial x^{2}}=\frac{\partial u}{\partial t}
$$

$$
V_{p}=\sqrt{\frac{E A}{\rho A}}=\sqrt{\frac{E}{\rho}}
$$

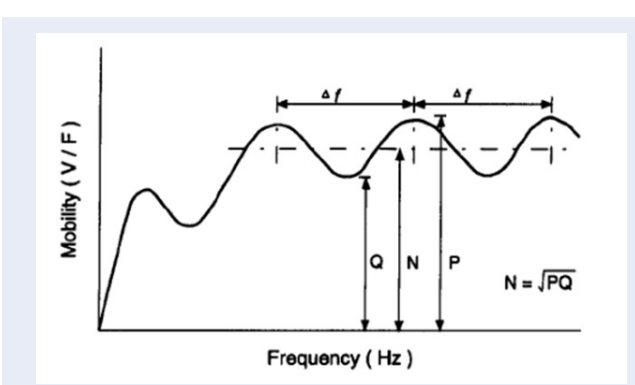

Figure 2: Mobility curve.

in which $\mathrm{V}_{p}$ is the wave velocity in the axial direction of the one-dimensional prismatic rod, $\mathrm{u}$ and $\mathrm{x}$ respectively is the displacement and coordinate in the axial direction; $\mathrm{E}, \rho$, and $\mathrm{A}$ is respectively the modulus of elasticity, mass density, and the cross-section of the rod.

The mechanical impedance or the reciprocation of the mobility of the structure is defined as below:

$$
Z=\frac{E A}{V} \alpha \frac{F}{V}
$$

in which $\mathrm{F}$ is the force applied to the structure in the axial direction. Any changes in $\mathrm{E}, \mathrm{A}$, or $\mathrm{V}$ due to defects, reduction/enlargement in cross-section, and low quality, etc. would result in a variation in the impedance. As such, this method of impedance is widely applicable in damage detection. With data analysis that is based partly on the maximum and minimum values of the pile mobility, the maximum and minimum area of the cross-section are computed $^{4}$.

\section{Method of the Transverse Wave Propagation}

An excitation would be applied to a specified point on the shaft of the structure and shear wave will travel within the structure body. This technique is also applicable to inelastic structure ${ }^{4}$, as in Figure 3below: By hearing using geophone for sonic sound, or seismic sensor mounting along the structure shaft, the response will be recorded at both the end of the structure; if there is a defect, the change in velocity amplitude will be found. 


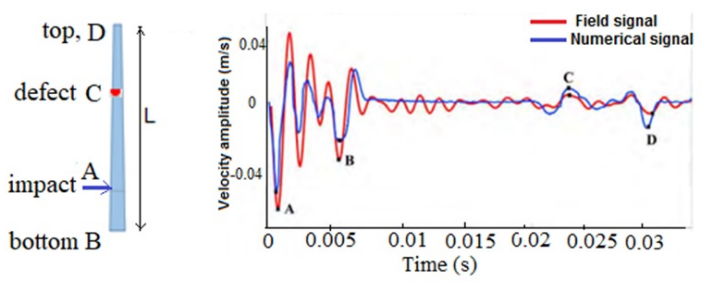

Figure 3: Shear wave propagation method ${ }^{5}$.

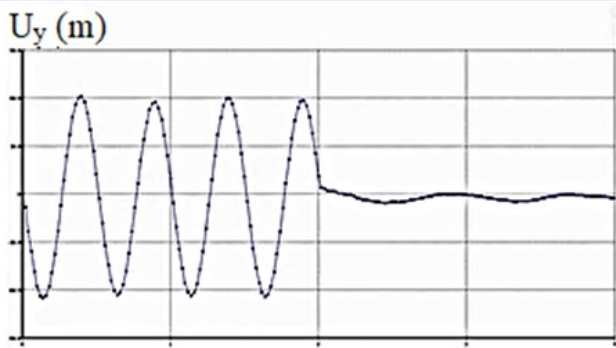

Dynamic time (s)

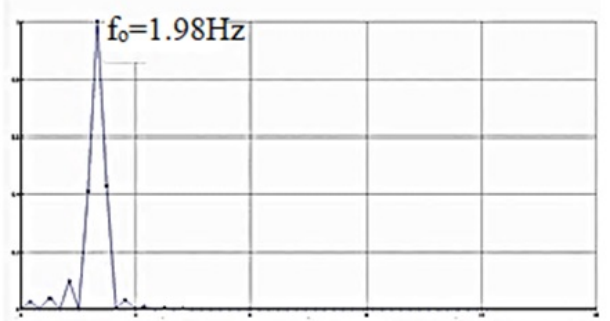

Frequency $(\mathrm{Hz})$

Figure 4: Signals in TD and their transformation into FD.

\section{Transforming algorithm to be used}

Fast Fourier Transform (FFT) is the traditional way to convert the time-domain signals to the frequencydomain response. Data in time-domain have $\mathrm{n}_{s}$ recordings, having the sampling frequency $\mathrm{f}_{s}$, which is the total time of sampling divided by $\mathrm{n}_{s}$. Nyquist frequency will be the $\mathrm{f}_{S} / 2$. The number of periods $\mathrm{np}_{f}$ during the time of sampling and the number of samples in a period will be $\mathrm{n}_{s} / \mathrm{np}_{f}$. As such, the frequency resolution in frequency domain analysis requires a sampling frequency $\mathrm{f}_{r e s}=1 / \mathrm{T}_{s}$. Time-domain recording will be at least $2^{n}$ data for being sufficient in FFT; the bigger amount of data is the more precise frequency spectrum is.

For the dynamic analysis, the load case would be of time history in a corporation with a dead load. A lateral excitation as a time-dependent loading $\mathrm{P}(\mathrm{t})=\mathrm{P}_{o}$. $\sin (2 \pi \mathrm{ft}+\theta)$ in which $\mathrm{f}$ is the excitation frequency in Hertz $(\mathrm{Hz})$. The excitation is an impact which applied in a very short duration (i.e., a few thousand seconds) to create a wave traveling along the column shaft. The response curves are of the signal in both time-domain (TD) and transformed into frequency-domain (FD) by the Fast Fourier Transformation (see Figure 4).

\section{Procedure for testing to be selected}

For checking the applicability of the Non-Destructive Test for soil-cement columns, two main tests are chosen as follows:

- Impact-on-column test

- Shear wave propagation

The former is a popular test for a pile in which the impedance of the structure will be computed. If there is any defect (i.e., necking, bulging. Low quality of material resulting in small modulus of stiffness) the cross area would be changed; or if there are some cracks or void, the reflectogram will display a pike in the middle time of wave travel. For the soil-cement column, the method is applied to check whether the impedance could be determined and changed or not. By using a numerical model in which some defects are intentionally created, the impact load is exerted on the column head. If the result cannot show any change in the impedance, the method is failed to apply to a semi-rigid structure.

The latter is the second alternative for assessing the change in the structure impedance. If an impact load is exerted at the column head or anywhere along the pile shaft, the velocity curve of every point on the individual sectors of the column would not be plotted; the method cannot be applied to the structure. 
As such, the former method uses the longitudinal Pwave traveling along the rod of the column, and the latter method uses the shear transversal S-wave to assess the integrity of the structure.

\section{MODEL}

\section{Model}

A single soil-cement $0.6 \mathrm{~m}$ diameter column is modeled as in Figure 5. Its material properties and crosssection are described in Table 1, but concerning the semi-rigid attributes of the structure, some modifications are tabulated as in Table 2. Springs in the column shaft are computed by Equations (4), (5), (6) and $(7)^{6}$. Spring stiffness and damping coefficient of the dashpot at the column tip, as in Figure 5are computed from a real project, based on the percentage of load delivered to the column ${ }^{7}$.

Soil properties are selected from a real site in Ho Chi Minh City. Main properties are described in detail of Table 2.

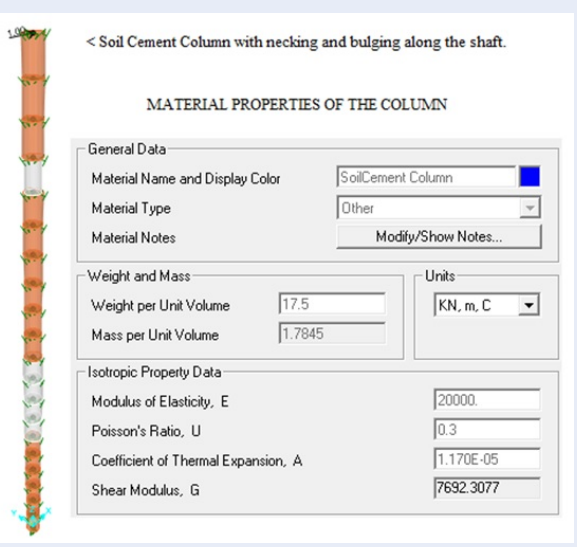

Figure 5: Defected column with lateral impact load at the head, with spring along the shaft and material properties.

For the pile mantle, the stiffness is

$$
\begin{gathered}
k_{v}=2.3 G_{s} \\
c_{v}=2 \pi \rho_{s} V_{s} d
\end{gathered}
$$

where $\mathrm{k}_{v} \mathrm{c}_{v}$ respectively is the vertical stiffness, and vertical damping component for the pile mantle; $\mathrm{V}_{S}$ is the shear wave velocity in the soil, $\mathrm{G}_{s}$ is the shear modulus of the soil. Both $\mathrm{k}_{v}$ and $\mathrm{c}_{v}$ are computed per unit length of the structure (i.e., $\mathrm{kN} / \mathrm{m} / \mathrm{m}$ and $\mathrm{kNs} / \mathrm{m} / \mathrm{m}$, respectively).
For the pile tip, the stiffness and damping are

$$
\begin{gathered}
K_{b}=\frac{4 G_{s} d}{\left(1-v_{s}\right)} \\
C_{b}=\frac{0.85 K_{b} d}{V_{s}}
\end{gathered}
$$

$\mathrm{K}_{b} \mathrm{C}_{b}$ respectively is the vertical stiffness and vertical damping component for the pile tip, $\rho_{s}$ is the soil bulk density, $v_{s}$ is the Poisson's ratio of the soil, $\mathrm{d}$ is the pile diameter;

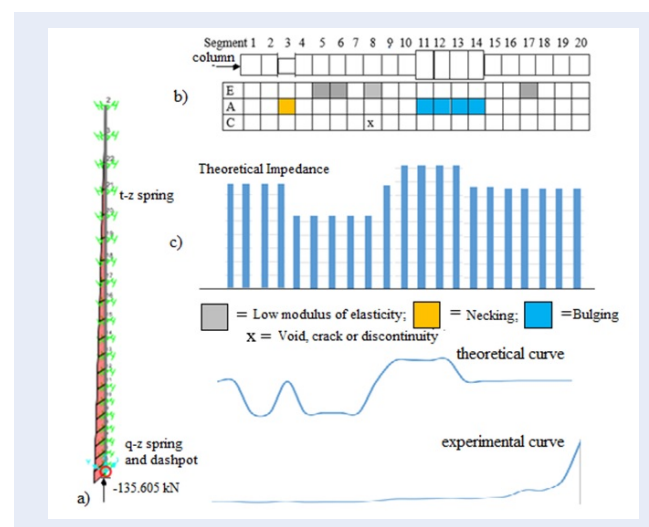

Figure 6: a) Model of the soil-cement column subjected to a vertical impact load; b) Scheme of defects; c) Impedance curve, computed theoretically and experimentally.

$\mathrm{G}_{s}$ is the shear modulus of the soil, $\mathrm{G}_{s}=\mathrm{E}_{S} / 2\left(1+v_{s}\right)$ with $\mathrm{E}_{S}$ is the modulus of elasticity of the soil.

The stiffness of skin friction spring will be assigned as the Link/support element in SAP $2000^{8}$, as illustrated in Figure 6a. In this study with a vertical impact load, for a practical purpose, the $\mathrm{V}_{s}$ shear wave velocity of the soft soil is computed by taking a modulus of elasticity $\mathrm{E}=12500 \mathrm{kN} / \mathrm{m}^{2}$, and $\mathrm{V}_{S}$ is $50-150 \mathrm{~m} / \mathrm{s}$. For plotting the time-domain response of the wave, we use the built-in tool of SAP 2000 software, in which the file will be converted into spectral velocity in frequencydomain by the Fast Fourier Transform (FFT) algorithm. The impedance curve is plotted from peak values at the dominant frequency $\mathrm{f}_{o}=1.15 \mathrm{~Hz}$ (Figure 7).

\section{Results}

Based on the material stiffness, cross-section and the density of the structure (i.e. mechanical impedance $\mathrm{Z}$ ), the theoretical curve of impedance has the shape as in Figure 6c.

For checking the applicability of the impact test, a transversal impact is also utilized. Time-domain 
Table 1: Properties of the column

\begin{tabular}{lll}
\hline Properties & Unit & Value \\
Modulus of elasticity & $\mathrm{kPa}$ & $2 \mathrm{e} 4$ \\
Bulging factor & - & 1.2 \\
Necking factor & - & 0.8 \\
Unit Weight & $\mathrm{kN} / \mathrm{m}^{3}$ & 17.5 \\
\hline
\end{tabular}

\section{Table 2: Soil Properties}

\begin{tabular}{llll}
\hline Properties & Unit & Layer $1^{*}$ & Layer 2 \\
$\gamma$ unsat $/ \gamma$ sat & $\mathrm{kN} / \mathrm{m}^{3}$ & $16 / 17$ & $18 / 20$ \\
Cohesion & $\mathrm{kPa}$ & 5 & 1 \\
Friction angle & $\mathrm{o}$ & 1 & 31 \\
Modulus of elasticity & $\mathrm{kPa}$ & $1.25 \mathrm{e} 4$ & $5 \mathrm{e} 4$ \\
Wave velocity & $\mathrm{m} / \mathrm{s}$ & $50-150$ & $<180$ \\
$\mathrm{SPT}$ & & 5 & 37 \\
\hline
\end{tabular}

${ }^{\star} 20 \mathrm{~m}$ of thickness.

(TD) signals and the spectral velocity in the frequency-domain (FD) of wave transmission in the axial direction are described in Figure 7. The impedance is plotted theoretically at the different locations of the column, as shown in Figure $6 c$, using the formula (3).

By analyzing the spectral velocity in the frequency domain, the velocity decreases from the column head (i.e., ground surface) to the tip, according to a parabolic trend at $\mathrm{R}^{2}=0.996$.

Although there are defects along the shaft of the column (as in Figure 6), no variation in the impedance of the semi-rigid column is clearly recognized, except the rapid trend of the increase of the impedance at the structure tip. The heterogeneous medium of the soilcement mixing might be the central reason for this. The impedance curve is quite different from that of the theoretical one (see Figure 8).

This implies that the semi-rigid structure like the soilcement column absorbs the vibration caused by the stress wave due to the impact load, no reflection from the bottom of the column found, and no indicator of the defects are detected. The impact method, both kinds of body waves such as longitudinal P-wave and transversal S-wave, may not be used to detect the defects in such a semi-rigid structure.

Although an impact with the amplitude $\mathrm{P}_{o}=10 \mathrm{kN}$ exerting at the column head in $\mathrm{x}$-direction and $\mathrm{z}$ direction, the impedance curve might be an increase in mobility from head to tip. Unlike the fast transmission of waves in the rigid structure of a reinforced
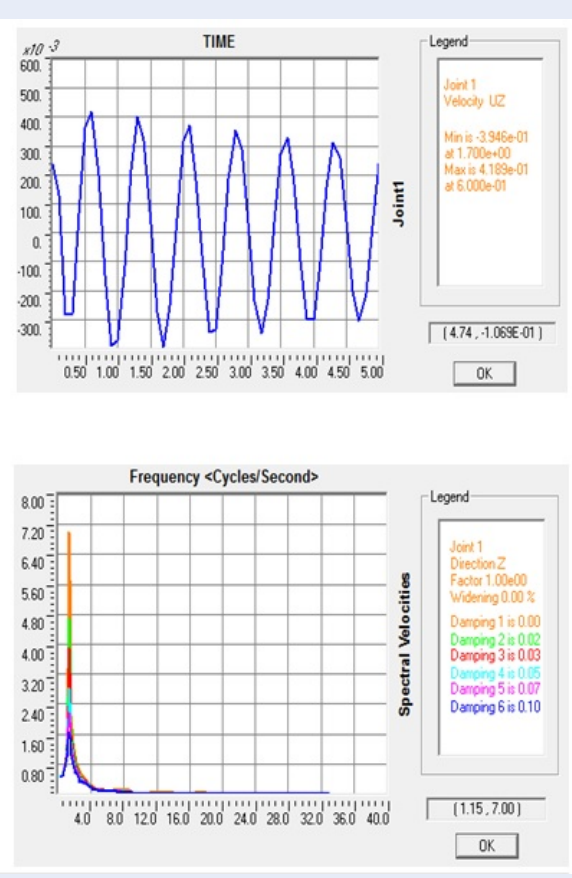

Figure 7: TD signals and frequency spectrum of the velocity at a specific location in the column body. 


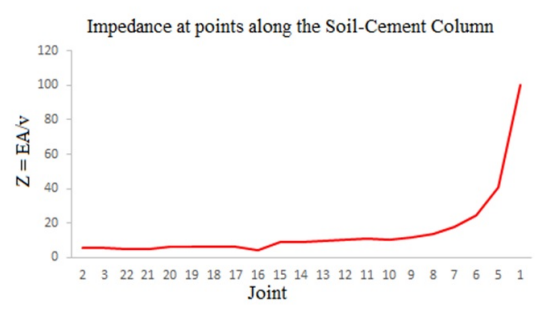

Figure 8: The impedance of the column from response velocity.

concrete pile, lateral impact in $\mathrm{x}$-direction causes the vibration that diminishes early at the depth of onefourth of the length of the structure and does not come to the column tip (Figure 8).

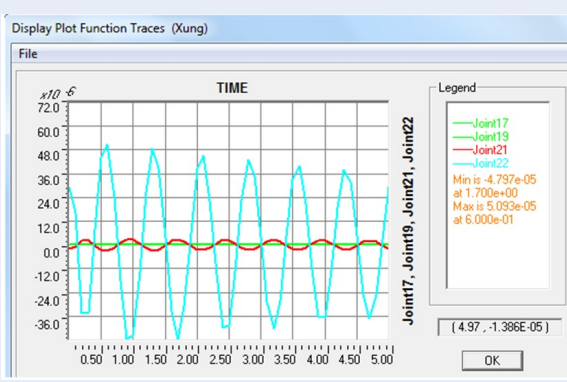

Figure 9: Responsive vibration shows the wave attenuation within the upper part of the column by SAP2000.

\section{DISCUSSION}

Some main issues required notation as follows:

- In the numerical model of a single column, spring stiffness and damping originated from the theory of elasticity. The assumption is not convincing on the semi-rigid structure, especially relating to the spring stiffness at pile tip ( $\mathrm{q}-\mathrm{z}$ spring) and pile shaft $(\mathrm{t}-\mathrm{z}$ spring). Nevertheless, the damping coefficient and spring stiffness are not too far as compared to prior research works ${ }^{2-4}$. These parameters strongly govern the analysis. As such, the formulas (4) to (7) should be tentatively studied from both theoretical and experimental approaches to be more reasonable.

- The impact load exerted on the column head causes a big deformation (displacement $\mathrm{U}_{y} \approx 0.1777 \mathrm{~m}, \mathrm{~V}_{y}$ $\approx 3,6 \mathrm{~m} / \mathrm{s}$ ). For the semi-rigid, this is definitely unsuitable to be viewed as a low-strain test with small deformation as commonly used in pile integrity tests or PIT. The stiffness is not so different than the structure could not be model a rigid body with spring and damper linked directly to the model. It might be a non-linear strain-stress relationship inside the structural material resulting in this incompatibility.

- The excitation is in the horizontal direction, whilst the impedance is computed via the amplitude of the spectral velocity in the vertical direction. This may be explained that there is a close relationship between the shear wave velocity and the longitudinal wave traveling along the shaft of the column. This is acceptable, at least in mathematical meaning.

- For studying 0.6 diameters 20-meter-long column with the modulus is about $2 \mathrm{e} 4 \mathrm{kPa}$, nearly equals to the modulus of the soil. Unlike the very rigid concrete pile in which the interaction is negligible, the interaction between a soil-cement column and the surrounding soil medium is remarkable, so there is no reflecting wave along the column shaft. The structure in the soil medium is not a bounded element. Nevertheless, the computed velocity by the numerical model with a finite element mesh of the soil medium is about 308 $\mathrm{m} / \mathrm{s}$, higher than that of the soil medium. This result is due to the higher stiffness of the column.

\section{CONCLUSION}

This study studies the wave propagating characteristics inside a soil-cement column to check the applicability of the vibration techniques in diagnosing the semi-rigid structure. The finite element model yields no detection against defects in the objects under study. The low quality of the material integrity, which is due to the heterogeneity from mixing the materials at the site, might be the main difficulty for applying the vibration techniques over the semi-rigid structure. The results indicate that the semi-rigid soilcement column with defects reflects no variation in the impedance; besides, the wave velocity traveling in semi-rigid is only hundreds meter per second, much lower than that in concrete material. The soil-cement column cannot be the same as the pile to name 'SoilCement Pile' as usual. Without the mechanism of load transmission to the surrounding soil, and based on the unclear variation in the impedance of the defected semi-rigid medium, it is disputable to apply the Non-Destructive Test, particularly the impedance method, to the soil-cement column. This study also agrees with the recommendation that it is necessary to integrate other different alternatives or methods of tests for this semi-rigid structure ${ }^{9}$.

\section{ABBREVIATIONS}

NDT: Non-Destructive Test

SHM: Structural Health Monitoring

PIT: Pile Integrity Test 
IL: Impedance Log Technique

CSL: Cross-hole Sonic Logging

TD: Time-domain; FD: Frequency-domain

FFT: Fast Fourier Transformation

\section{COMPETING INTERESTS}

The author ensures that there is no conflict of interest in publishing this article.

\section{AUTHORS' CONTRIBUTIONS}

Tham Hong Duong is the author who owns all the ideas for the article, collects data and analyzes the results obtained, and prepares the manuscript in English.

\section{REFERENCES}

1. Lai JR, Yu CP, Liao ST. Assessment of the Integrity of Piles by Impedance Log Technique. Paper. 2006;Available from: https: //doi.org/10.4028/www.scientific.net/KEM.321-323.340.

2. Varosio G. A Non-Destructive Testing Program for a Group of Jet Grouting Columns, presented at the 4th International Conference on Case Histories in Geotechnical
Engineering, Missouri University of Science and Technology. 1998;Available from: https://scholarsmine.mst.edu/icchge/ 4icchge/4icchge-session07/10.

3. Liao ST, Tong $\mathrm{JH}, \mathrm{Chen} \mathrm{CH}$, Wu TT. Numerical simulation and Experimental Study of Parallel Seismic Tests for Pile. International Journal of Solids and Structures. 2006;43:2279-2298. Available from: https://doi.org/10.1016/j.ijsolstr.2005.03.057.

4. Varma SJ, Gopalakrishnan N, Kumar KS, Sakaria PE. Structural Integrity Evaluation of Pile Foundations by Pile Integrity Testing. International Journal of Structural and Civil Engineering Research. 2013;2(3):133-140.

5. Pat Rajeev: Smart Monitoring for Condition Assessment of InfraStructure, presented at the International Conference in Recent Trends in Geotechnical Engineering and Education, Brisbane, Australia. 2020;

6. Mladen C, Boris F, Radomia F. Numerical Simulation of The Pile Integrity Test on Defected Piles;Available from: https://www. researchgate.net/publication/279080437.

7. Tien NT. Dynamic and static behavior of driven piles. Swedish Geotechnical Institute, Report No3, Einkoping. 1987;p. 26-197.

8. Sap2000 User Manual;Available from: https://www.civilax.org/ sap2000-manual.

9. Ryden N, Ekdahl U, Lindh P. Quality control of Cement Stabilised Soil using Non-Destructive Seismic Test, Lecture 34, presented at the Conference on Advanced Testing of Fresh Cementitious Materials. 2006;Available from: https:// www.researchgate.net/publication/259569172. 